This document is the accepted manuscript version of the following article:

Trotochaud, L., Head, A. R., Pletincx, S., Karslıoğlu, 0., Yu, Y., Waldner, A., ...

Bluhm, H. (2018). Water adsorption and dissociation on polycrystalline copper oxides:

effects of environmental contamination and experimental protocol. Journal of physical

Chemistry B, 122(2), 1000-1008. https://doi.org/10.1021/acs.jpcb.7b10732

\title{
Water Adsorption and Dissociation on Polycrystalline Copper Oxides: Effects of Environmental Contamination and Experimental Protocol
}

\author{
Lena Trotochaud, ${ }^{l}$ Ashley R. Head, ${ }^{1}$ Sven Pletincx, ${ }^{1,2}$ Osman Karslıoğlu, ${ }^{l} \quad Y i \quad Y u,{ }^{1,3}$ \\ Astrid Waldner, ${ }^{1,4}$ Line Kyhl, ${ }^{1,5}$ Tom Hauffman, ${ }^{2}$ Herman Terryn, ${ }^{2}$ Bryan Eichhorn, ${ }^{3}$ \\ and Hendrik Bluhm ${ }^{1,6}$
}

Correspondence should be addressed to H.B.

hbluhm@lbl.gov

${ }^{1}$ Chemical Sciences Division, Lawrence Berkeley National Laboratory, Berkeley, CA, 94720 USA

${ }^{2}$ SURF Research Group, Department of Materials and Chemistry, Vrije Universiteit Brussel, Pleinlaan 2, 1050 Brussels, Belgium

${ }^{3}$ Department of Chemistry and Biochemistry, University of Maryland, College Park, MD, 20742 USA

${ }^{4}$ Laboratory of Environmental Chemistry, Paul Scherrer Institute, 5232 Villigen PSI, Switzerland

${ }^{5}$ Department of Physics and Astronomy and Interdisciplinary Nanoscience Center, Aarhus University, Aarhus C DK-8000, Denmark

${ }^{6}$ Advanced Light Source, Lawrence Berkeley National Laboratory, Berkeley, CA, 94720 USA 


\begin{abstract}
We use ambient pressure X-ray photoelectron spectroscopy (APXPS) to study chemical changes, including hydroxylation and water adsorption, at copper oxide surfaces from ultra-high vacuum to ambient relative humidities of $\sim 5 \%$. Polycrystalline $\mathrm{CuO}$ and $\mathrm{Cu}_{2} \mathrm{O}$ surfaces were prepared by selective oxidation of metallic copper foils. For both oxides, hydroxylation occurs readily, even at high vacuum conditions. Hydroxylation on both oxides plateaus near $\sim 0.01 \%$ relative humidity $(\mathrm{RH})$ at a coverage of $\sim 1$ monolayer. In contrast to previous studies, neither oxide shows significant accumulation of molecular water; rather, both surfaces show a high affinity for adventitious carbon contaminants. Results of isobaric and isothermic experiments are compared, and the strengths and potential drawbacks of each method are discussed. We also provide critical evaluations of the effects of the hot filament of the ion pressure gauge on the reactivity of gasphase species, the peak fitting procedure on the quantitative analysis of spectra, and rigorous accounting of carbon contamination on data analysis and interpretation. This work underscores the importance of considering experimental design and data analysis protocols during APXPS experiments with water vapor in order to minimize misinterpretations arising from these factors.
\end{abstract}




\section{$1 \quad$ Introduction}

The interaction between water and metal oxide surfaces is an important process in a wide range of environmental and atmospheric systems, industrial heterogeneous catalysis, and corrosion. One of the most intriguing questions is the influence of hydroxylation and molecular water adsorption on the reaction of metal oxide surfaces with other trace gases. The chemical effects of ambient water are of particular interest for the performance of filtration materials for protection against chemical warfare agents and other toxic gases. ${ }^{1-3}$ Metals, metal salts, and/or metal oxides are components of commonly utilized filtration materials for absorption and degradation of various toxic organic compounds. ${ }^{4}$ Changes in the chemical environment of metalcontaining surfaces by exposure to common atmospheric components (e.g. water) may affect the adsorption and eventual decomposition mechanisms of toxic molecules in filtration materials. The effects of relative humidity on filter performance are not well understood, and water will certainly be present during filter storage (from the ambient environment) and use (from respiration).

Ambient pressure X-ray photoelectron spectroscopy (APXPS) is an excellent method to investigate the reaction of water vapor with metal oxides and the influence of hydroxylation and water adsorption of the metal oxide surface on the reaction with trace gases. The particular strength of APXPS is that lattice oxygen, hydroxyl groups, and adsorbed molecular water can, in most cases, be easily distinguished from each other in $\mathrm{O} 1 \mathrm{~s}$ spectra due to differences in their chemical shifts. While APXPS experiments at 1 atm were recently demonstrated, ${ }^{5,6}$ they are still far from routine due to the strong scattering of electrons at atmospheric pressures and/or the need for the use of specially designed and fabricated isolated gas-filled experimental cells which use ultrathin membranes; such constrains greatly hamper, at the time of this writing, the study of a broad range of samples. Fortunately, the need for specialized equipment to approach $1 \mathrm{~atm}$ is not a requirement 
in measurements that are solely concerned with the influence of water vapor and trace gases on the surface chemistry of the material under investigation. The saturation water vapor pressure at room temperature is about 20 Torr, and the partial pressures of the trace gases in the environment (e.g., $\mathrm{CO}_{\mathrm{x}}, \mathrm{NO}_{\mathrm{x}}, \mathrm{SO}_{\mathrm{x}}, \mathrm{O}_{3}$ ) is much lower, with the $\mathrm{CO}_{2}$ pressure as the highest with about 0.4 Torr. These are conditions that can now be achieved fairly routinely in APXPS experiments.

APXPS has been used over the past decade to study water interaction with a wide range of metal oxides, both on single crystals and epitaxial films, such as $\mathrm{SiO}_{2} / \mathrm{Si}(111),{ }^{7}$ rutile $\mathrm{TiO}_{2}(110),{ }^{8}$ $\mathrm{LaFeO}_{3} / \mathrm{SrTiO}_{3}(001),{ }^{9} \mathrm{LaCoO}_{3}(001),{ }^{10} \mathrm{FeO} / \mathrm{Au}(111),{ }^{11} \alpha-\mathrm{Fe}_{2} \mathrm{O}_{3}(0001),{ }^{12} \alpha-\mathrm{Fe}_{2} \mathrm{O}_{3}$ on $\mathrm{Au}(111),{ }^{13}$ $\mathrm{Fe}_{3} \mathrm{O}_{4}(001),{ }^{14} \mathrm{Al}_{2} \mathrm{O}_{3} / \mathrm{NiAl}(110),{ }^{15} \mathrm{Sm}$-doped $\mathrm{CeO}_{2}(100),{ }^{16}$ and $\mathrm{MgO}(100),{ }^{17,18}$ as well as polycrystalline samples, e.g. La-based perovskites, ${ }^{19}$ anatase $\mathrm{TiO}_{2},{ }^{20} \mathrm{GeO}_{2} / \mathrm{Ge}(100),{ }^{21} \mathrm{Cu}_{2} \mathrm{O},{ }^{22-24}$ and $\mathrm{Al}_{2} \mathrm{O}_{3}{ }^{22}$. These studies have shown that the onset and extent of surface hydroxylation depends both on the orientation of the crystal face as well as the number of surface defects; polycrystalline surfaces show in general a lower onset for hydroxylation than single crystalline samples. An intriguing finding is that most single-crystalline metal oxide surfaces show a common onset humidity of $0.01 \%$ for terrace (i.e., non-defect) hydroxylation. While the precise mechanism for this behavior is not yet understood, theoretical studies indicate that dissociation of an isolated water molecule is kinetically hindered, while dissociation of a water molecule that forms a complex with other adsorbed water molecules, as well as lattice oxygen, is more facile..$^{25,26}$ According to this picture, a relative humidity $(\mathrm{RH})$ of $0.01 \%$ is the critical onset value for the formation of these complexes; below this $\mathrm{RH}$, the time-averaged number of water molecules at the metal oxide surface is not large enough to lead to complex nucleation. Since the hydroxyl-water bond is stronger than the water-water bond, adsorption of water molecules proceeds as soon as hydroxyl 
groups have formed on the surface, which has been observed in APXPS measurements demonstrating that water adsorption goes hand-in-hand with hydroxylation.

While studies of water dissociation and adsorption on metal oxide surfaces are becoming more common and currently can be considered "routine" in the APXPS community, standard experimental protocols can vary considerably depending on the research group and the class of materials studied. Isobaric and isothermic experiments are both standard practice, but the relative humidity range accessible depends on the sample preparation requirements and thus the type of sample holders that can be utilized. In isobaric experiments, the relative humidity is controlled by changing the sample temperature. For example, relative humidity values approaching $100 \%$ can only be attained currently by using a sample stage that can be cooled to below the temperature of the surrounding surfaces during measurements, which often leads to enhanced adsorption of carbonaceous contaminants on the sample. In general, isobaric studies from low to high RH are the cleanest experiments, since the sample is initially kept at elevated temperatures (typically several hundred ${ }^{\circ} \mathrm{C}$ ) where it remains clean. The sample is then cooled down in the presence of mTorr to Torr of water vapor, in which molecular $\mathrm{H}_{2} \mathrm{O}$ represents by far the majority gas phase species and thus is favored as an adsorbate over other contaminants with lower partial pressures. Isobaric studies have the drawback that the gas phase and the sample surface temperatures are not the same, which can lead to slight uncertainties in the calculation of the RH. ${ }^{12}$ However, isobaric water uptake experiments on $\mathrm{MgO}(100)$ over a pressure range of three orders of magnitude have shown distinct differences in the onset temperature for terrace hydroxylation and water uptake which, when converted to RH, coincided with each other (within the error bars), showing that the temperature effect in isobar experiments is most likely negligible. ${ }^{17}$ In isotherm experiments from low to high water partial pressure (and thus $\mathrm{RH}$ ), the partial pressure of water in the low RH regime 
is comparable to that of background contaminants which often leads to increased adsorption of carbonaceous species in these experiments.

The presence of carbon contaminants in APXPS experiments with water vapor can have unintended effects which will manifest in the measured data. Surface carbon contamination cannot be assumed to be only a spectator, as is often done with UHV XPS measurements. (This assumption is so common that the aliphatic adventitious carbon is often used for binding energy calibration.) Oxidized carbonaceous species are often inherently hydrophilic, and thus it is reasonable to expect the identity of the surface carbon contamination could influence the interaction of gas-phase water with metal oxide surfaces. In any case, the possible effects of surface carbon on surface wetting and reactivity certainly cannot be assessed if $\mathrm{C} 1 \mathrm{~s}$ spectra are not collected and carefully analyzed.

For the correct interpretation of $\mathrm{O}$ 1s spectra, which contain most information on the reaction of water with the surface, it is imperative to identify oxidized surface carbon species, which will obviously contribute features to the O 1s spectra. A powerful strength of XPS is the ability to quantitatively analyze the spectra by comparing peak areas of different species, provided that relative sensitivity factors are determined when quantifying across different core levels. Jribi et al. demonstrate the utility of identification and quantification of surface carbon contaminants in studying the intrinsic hydrophilicity of $\mathrm{TiO}_{2}{ }^{20}$ Stoerzinger et al. have provided a rigorous accounting of surface carbon contamination in their experiments of water vapor at perovskite surfaces. ${ }^{9,10,19}$ In those works, surface hydroxylation preceded formation of surface carbonates, which displaced hydroxyl groups prior to molecular water adsorption. ${ }^{10}$ The surface carbonate coverage was found to increase with increasing relative humidity (isobar experiments at 0.1 Torr water vapor pressure), reaching a surface coverage of $0.2-0.3$ monolayers at $0.1 \% \mathrm{RH}$. For these 
materials, where the catalytic activity for water splitting was found to be intimately related to the surface termination and wetting, ${ }^{9,19}$ these studies demonstrate that the influence of surface carbon species on the desired material properties cannot be ignored.

Cracking of gas-phase species over the hot ion gauge filament (typically thoria/yttriacoated iridium or tungsten) during APXPS experiments, while certainly possible, is rarely mentioned in the literature. Cracking of gas molecules over hot metals is also used intentionally in surface science studies in the production of atomic oxygen and hydrogen. In the case of water, cracking may facilitate surface hydroxylation at low water vapor pressures, although the prevalence and extent of this process over different surfaces is currently unknown.

Here, we will discuss some of these practical considerations of APXPS experiments with water vapor on polycrystalline $\mathrm{CuO}$ and $\mathrm{Cu}_{2} \mathrm{O}$, which provides an exemplary case study in the importance of considering experimental design and data analysis protocols in similar studies. In general, the copper oxides follow the trends observed on other metal oxide surfaces upon exposure to water vapor; namely, hydroxylation occurs readily at low relative humidities, and the surface hydroxyl coverage reaches $\sim 1$ monolayer when molecular water adsorption is observed. On both oxides, surface carbon contamination coverage increases with increasing relative humidity and accounts for most of the changes observed in the $\mathrm{O} 1 \mathrm{~s}$ spectra. We also observe partial reduction of $\mathrm{CuO}$ upon water vapor exposure when the ion gauge is left on during the APXPS measurement at low water vapor pressures. Furthermore, surface hydroxylation appears to be facilitated on both $\mathrm{CuO}$ and $\mathrm{Cu}_{2} \mathrm{O}$ with the ion gauge turned on. 


\section{Experimental}

2.1 Ambient-pressure X-ray photoelectron and near-edge X-ray absorption fine-structure spectroscopy.

XPS and NEXAFS experiments were performed in the ambient-pressure XPS (APXPS-1) $)^{27}$ endstation at beamline 11.0.2 $2^{28}$ at the Advanced Light Source at Lawrence Berkeley National Laboratory. Milli-Q purified water $(18.2 \mathrm{M} \Omega \mathrm{cm})$ was mounted onto the APXPS analysis chamber in a glass vessel, degassed by three cycles of liquid nitrogen freeze-pump-thaw, and introduced into the XPS analysis chamber via a high-precision leak valve. The base pressure of the analysis chamber was $1 \times 10^{-8}$ Torr or better for each experiment. Prior to moving the sample into the analysis chamber, the water leak valve was calibrated so that desired water vapor pressures below $1 \times 10^{-3}$ Torr could be accessed with the ion pressure gauge turned off; this was accomplished by recording the number of turns opening the leak valve from the fully closed position that were required to reach a desired pressure. Calibration values were checked at least twice before each experiment to ensure reproducibility. Pressures of $1 \times 10^{-3}$ Torr and higher were monitored with a 10 Torr capacitance manometer (Baratron $\left.{ }^{\circledR}\right)$.

A ceramic heater embedded in the sample holder was used to heat the samples during oxide preparation and isobar measurements. The binding energy scale for XPS spectra was calibrated to the position of the $\mathrm{Cu} 2 p_{3 / 2}$ main peak ( $932.9 \mathrm{eV}$ for $\mathrm{CuO}^{2}$ and $932.4 \mathrm{eV}$ for $\mathrm{Cu}_{2} \mathrm{O}^{29}$ ). Spectra were analyzed with the KolXPD software package, using Voigt lineshapes and Shirley or linear backgrounds. More detailed information about XPS fitting procedures can be found in the Supporting Information. Spectra presented in the main text are from representative experiments where the carbon contamination was relatively low, however, isobar and isotherm experiments on both $\mathrm{CuO}$ and $\mathrm{Cu}_{2} \mathrm{O}$ surfaces were performed at least three times each to confirm reproducibility. 
To prevent beam-induced changes to the sample surface, the beam spot was defocused $(\sim 200 \times 200$ $\mu \mathrm{m}^{2}$ ), its position on the sample was changed every 2-4 minutes (after collecting 2-4 spectra), and the X-ray beam was blocked with a shutter between measurements. On the timescale of the measurements presented here ( $\sim 3-4$ min at each spot), no evidence of beam-induced changes was observed.

\subsection{Preparation of oxide surfaces.}

$\mathrm{CuO}$ and $\mathrm{Cu}_{2} \mathrm{O}$ samples were prepared from high purity metal foils $(\mathrm{Cu}: 99.999 \%$ metals basis, Alfa Aesar, $0.5 \mathrm{~mm}$ thick). The $\mathrm{Cu}$ foil was cut into pieces $\sim 1.5 \mathrm{~cm}$ in diameter and precleaned by sequential sonication in acetone, isopropanol, and ethanol (20 min each), followed by blow-drying with nitrogen. Pre-cleaned $\mathrm{Cu}$ foils were oxidized in air, at $450{ }^{\circ} \mathrm{C}$ for $\mathrm{CuO}$ and $300{ }^{\circ} \mathrm{C}$ for $\mathrm{Cu}_{2} \mathrm{O}$, for $30 \mathrm{~min}$, followed by sonication in ethanol. This treatment was repeated, giving a total of two oxidation-sonication cycles per sample. The foils were then treated further in the endstation sample preparation chamber to generate a single copper oxidation state. For $\mathrm{CuO}$, samples were heated in 1 Torr $\mathrm{O}_{2}$ for at least 30 min to remove adventitious carbon contamination and fully oxidize any $\mathrm{Cu}_{2} \mathrm{O}$ present at the surface, cooled to $\sim 200^{\circ} \mathrm{C}$ in $1 \mathrm{Torr} \mathrm{O}_{2}$, and then further cooled under vacuum to room temperature $\left(\sim 20^{\circ} \mathrm{C}\right)$ before being transferred to the APXPS analysis chamber. For $\mathrm{Cu}_{2} \mathrm{O}$, the samples were heated under vacuum $\left(<1 \times 10^{-8}\right.$ Torr $)$ for at least 30 min to remove adventitious carbon contamination and to reduce any $\mathrm{CuO}$, then cooled to room temperature $\left(20{ }^{\circ} \mathrm{C}\right)$ in the preparation chamber before being transferred to the APXPS analysis chamber. The $\mathrm{Cu}$ oxidation state of the samples was confirmed by a combination of $\mathrm{Cu} 2 \mathrm{p}_{3 / 2}$ XPS and $\mathrm{Cu}$ L-edge NEXAFS, as described in our previous publication. ${ }^{2}$ (See Supporting Information for these spectra.) 


\section{$3 \quad$ Results and Discussion}

\subsection{Fitting of APXPS spectra and identification of surface species.}

Representative XPS data for isotherm $\left(20^{\circ} \mathrm{C}\right)$ experiments with $\mathrm{CuO}$ and $\mathrm{Cu}_{2} \mathrm{O}$ are shown in Figure 1. The spectra are normalized on the y-axis to the oxygen lattice peak $\mathrm{O}_{\text {lat }}$, near $529.9 \mathrm{eV}$ and $530.4 \mathrm{eV}$ for $\mathrm{CuO}$ and $\mathrm{Cu}_{2} \mathrm{O}$, respectively, and a Shirley-type background has been subtracted from each. Relative to the metal oxide peak, increasing intensity is observed with increasing water vapor pressure in the binding energy region between $531-534 \mathrm{eV}$, associated with an increase in $\mathrm{OH}$, carbonaceous $\left(\mathrm{C}-\mathrm{O}_{\mathrm{x}}\right)$, and molecular water species. At higher relative humidity values, a peak for water vapor $\left(\mathrm{H}_{2} \mathrm{O}_{(\mathrm{v})}\right)$ is observed above $535 \mathrm{eV}$. In the $\mathrm{Cu}_{2} \mathrm{O}$ spectra, a small peak near $529 \mathrm{eV}$ is also observed. This peak has been previously identified as either adsorbed oxygen on the $\mathrm{Cu}_{2} \mathrm{O}$ surface, subsurface oxygen, or some other surface defect. ${ }^{22-24}$ We note that the binding energy position of this peak is very similar to that of $\mathrm{CuO}$; however, we do not see any evidence in the $\mathrm{Cu} 2 \mathrm{p}_{3 / 2}$, valence band, or Cu L-edge NEXAFS spectra to suggest that a significant population of $\mathrm{Cu}^{2+}$ sites is present (see spectra in the Supporting Information). This peak decreases with increasing water vapor pressure, which could indicate either that these sites are reacting with surface adsorbates and are being consumed or that they are simply being covered by surface adsorbates. Spectra collected during isobar $(0.1$ Torr $)$ experiments appear similar, although typically with lower intensity in the region associated with carbonaceous species, as heating the surface can decrease adsorption of carbon contaminants. No changes were observed in the $\mathrm{Cu} 2 \mathrm{p}_{3 / 2}$ or $\mathrm{Cu}$ L-edge NEXAFS spectra on either surface upon water exposure (see Supporting Information). 

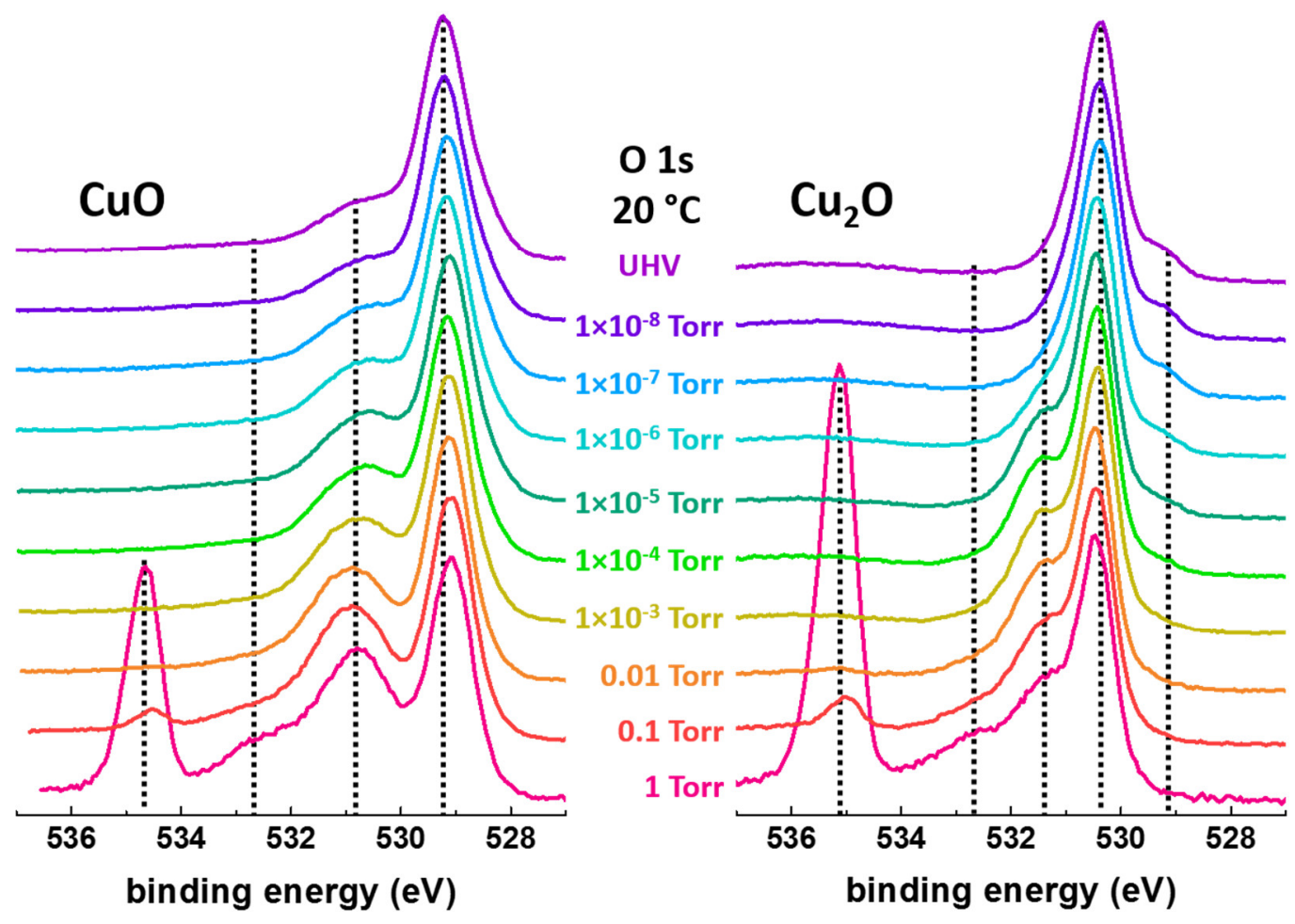

Figure 1. $\mathrm{O} 1 s$ spectra for $\mathrm{CuO}$ (left) and $\mathrm{Cu}_{2} \mathrm{O}$ (right) samples exposed to increasing water vapor pressures (at $20^{\circ} \mathrm{C}$ ) from UHV conditions $\left(<1 \times 10^{-8}\right.$ Torr) up to $\sim 6 \% \mathrm{RH}$. Dotted vertical lines behind the data are guides for the eye to highlight changing intensities. Spectra are normalized to the $\mathrm{CuO}$ or $\mathrm{Cu}_{2} \mathrm{O}$ oxygen lattice peaks, and the binding energy scales are calibrated to the position of the $\mathrm{Cu} 2 p_{3 / 2}$ main peak.

Representative fits of the isotherm data at a water vapor pressure of 0.1 Torr are shown in Figure 2. In the case of $\mathrm{Cu}_{2} \mathrm{O}$, a feature at higher binding energies near the background is present in all spectra (see Figure 1) and is shown in the Figure 2 fit as a dashed curve. In our spectra, this feature is fit well by fixing the binding energy difference between it and the main $\mathrm{Cu}_{2} \mathrm{O}$ oxide peak, as well as the relative areas of these two peaks. This feature has been observed previously, ${ }^{29,30}$ but to the best of our knowledge, its origin has not been discussed in detail. (It is also at the high-end of the range where $\mathrm{O} 1 \mathrm{~s}$ spectra are typically collected, and thus may often be overlooked. In this study, we have extended the collection window to include the feature for gas- 
phase $\mathrm{H}_{2} \mathrm{O}$.) The position and intensity relative to the main oxide peak could be consistent with a shake-up excitation of an electron in the final-state from the conduction band to the valence band; similar $\mathrm{O} 1 \mathrm{~s}$ features are commonly observed in polymers arising from HOMO $\rightarrow$ LUMO finalstate excitations. ${ }^{31}$

The remaining features in the $\mathrm{O} 1 \mathrm{~s}$ spectra are labeled in Figure 2 and correspond to the oxide lattice atoms $\mathrm{O}_{\text {lat, }}$, surface hydroxyl groups $\mathrm{OH}$, and surface carbon contamination $\mathrm{C}-\mathrm{O}_{\mathrm{x}}$. During fitting, the area of the $\mathrm{C}-\mathrm{O}_{\mathrm{x}}$ peak was constrained based on the area of the $\mathrm{C} 1 \mathrm{~s}$ peaks corresponding to oxidized carbon species and relative sensitivity factors calculated from measurement of gas-phase $\mathrm{CO}_{2}$. The protocol for fitting the $\mathrm{C}-\mathrm{O}_{\mathrm{x}}$ peak will be discussed in more detail below. As shown in Figure 2, at room temperature and a water vapor pressure of 0.1 Torr, the spectra are well fit with only these species and there is no obvious chemical signature for molecular water, which is typically expected between $532-534 \mathrm{eV}$ (i.e. between the surface $\mathrm{OH}$ peak and the gas-phase water peak). The $\mathrm{C}-\mathrm{O}_{\mathrm{x}}$ peak would appear to be perhaps a misidentified $\mathrm{H}_{2} \mathrm{O}$ peak, but as we will explain below, this assignment is not the case for these measurements.
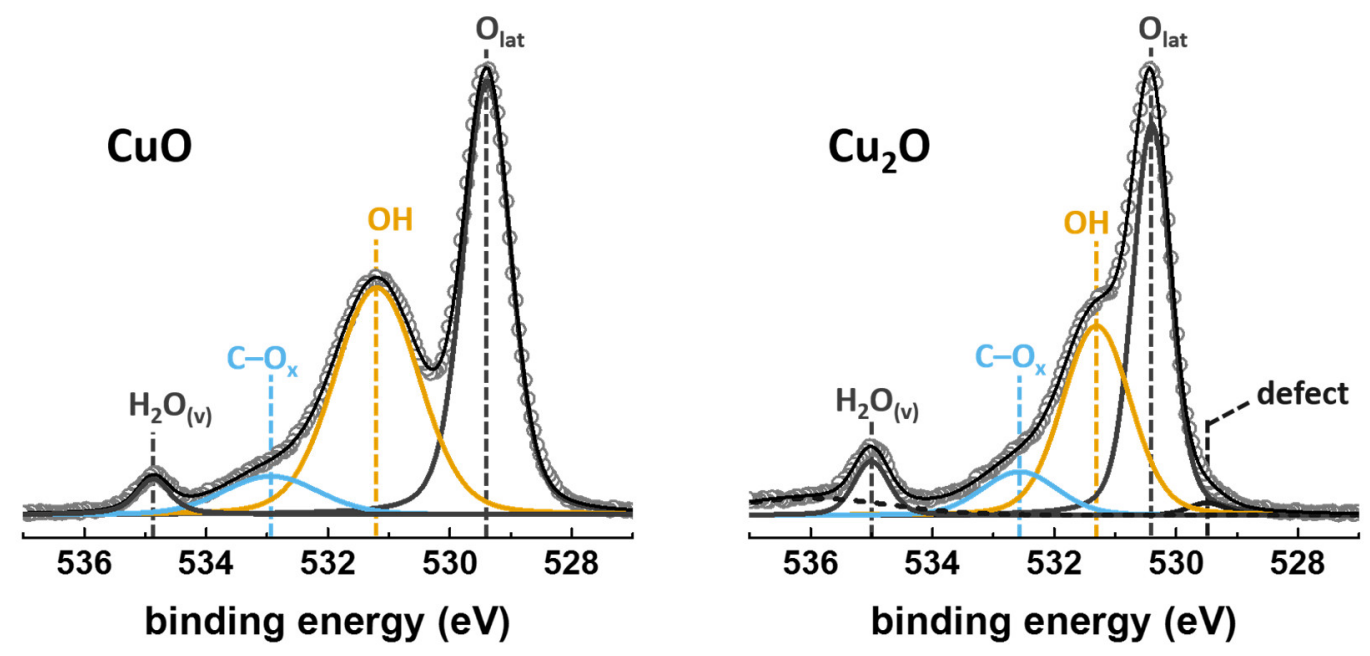

Figure 2. Representative fits for $\mathrm{O} 1 s$ spectra under 0.1 Torr water vapor pressure at $20{ }^{\circ} \mathrm{C}(\sim 0.001 \% \mathrm{RH})$ for $\mathrm{Cu}_{2} \mathrm{O}$ (left) and $\mathrm{CuO}$ (right). Fitted peaks for individual species are shown with thick solid lines, the sum of the fitted peaks is shown as a thin solid black line, and the data points are shown as grey circles. The small peak in the $\mathrm{Cu}_{2} \mathrm{O}$ spectrum shown as a black dashed curve represents a component present in all 
spectra that scales with the $\mathrm{O}_{\text {lat }}$ intensity and likely represents a shake-up feature from the $\mathrm{O}_{\text {lat }}$ peak. More information can be found in the Supporting Information file.

We will now briefly explain the protocol for including the $\mathrm{C}-\mathrm{O}_{\mathrm{x}}$ peak shown in Figure 2 . In the case of the copper oxide surfaces studied here, the quantification of adventitious surface carbon is a point which cannot be ignored. Figure 3 shows the $\mathrm{C} 1 \mathrm{~s}$ spectra collected immediately after the $\mathrm{O} 1 \mathrm{~s}$ spectra plotted in Figure 2. On both surfaces, the spectra are fit well by three peaks. The binding energy values for the different peaks show that the majority of the surface carbon contamination is in an oxidized state (peaks near $288 \mathrm{eV}$ and $287 \mathrm{eV}$ ), with a small population of aliphatic $\mathrm{C}-\mathrm{H}$ species (near $285 \mathrm{eV}$ ). These spectra are representative of the carbon species observed across multiple experiments performed across several experimental runs, spanning more than half a year. Presumably, the adventitious carbon species in the chamber background are relatively constant throughout any one day during a measurement. The consistency in the appearance of the $\mathrm{C}$ 1s spectra across samples and experimental runs, which all show that the peak near $287 \mathrm{eV}$ is smaller for $\mathrm{Cu}_{2} \mathrm{O}$ relative to $\mathrm{CuO}$, suggests that there is some difference in either affinity or reactivity of certain carbon species between the two oxide surfaces.

There are multiple different carbon-containing moieties which typically fall in the binding energy region between 287-288 eV, including various carbonyl, carboxyl, ether, and alcohol groups. ${ }^{31}$ Since the $\mathrm{O} 1 \mathrm{~s}$ spectrum is dominated by signal originating from the oxide substrate, conclusive identification of the surface carbon species on each surface is not possible only by APXPS. We can however draw some general conclusions about these carbon-containing species; namely, they likely contain between one and two oxygen atoms per carbon atom, and the corresponding O 1s peaks likely occur in the region between 532-534 eV. (See the Supporting Information for more detail.) These general guides are helpful in deciding how to appropriately place and constrain the $\mathrm{C}-\mathrm{O}_{\mathrm{x}}$ peak during fitting of the $\mathrm{O} 1 \mathrm{~s}$ spectra. 

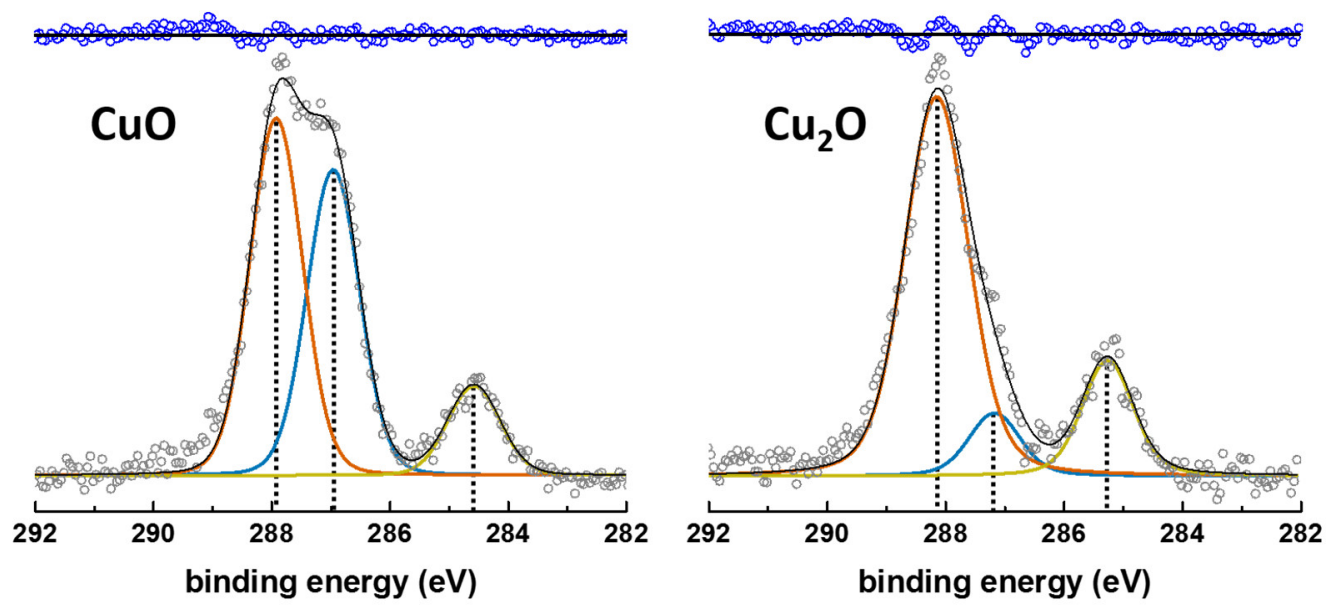

Figure 3. C 1s spectra taken immediately after the $\mathrm{O} 1 \mathrm{~s}$ spectra shown in Figure 2 (at $20^{\circ} \mathrm{C}$ and 0.1 Torr water vapor pressure). Peaks for oxidized carbon species are observed near $287 \mathrm{eV}$ and $288 \mathrm{eV}$ (blue and orange, respectively) and aliphatic carbon appears near $285 \mathrm{eV}$ (gold). Fitted peaks for individual species are shown with thick solid lines, the sum of the fitted peaks is shown as a thin solid black line, and the data points are shown as grey circles. Difference spectra between the data points and the total fit are shown at the same scale in blue at the top.

Figure 4 compares several fits of a single $\mathrm{O} 1 \mathrm{~s}$ spectrum collected for $\mathrm{CuO}$ at $20{ }^{\circ} \mathrm{C}$ under 0.1 Torr of water vapor. In each fit, different parameters were used for fitting the $\mathrm{C}-\mathrm{O}_{\mathrm{x}}$ peak and, when necessary, an additional peak for adsorbed molecular water was included. In Figure 4a, no peak is included for $\mathrm{C}-\mathrm{O}_{\mathrm{x}}$ and the water peak increases in intensity to fit this part of the spectrum. This fit gives a peak position for water of $533.8 \mathrm{eV}$, which is similar to that reported by Deng et al. where no peak was included for carbonaceous species during fitting. ${ }^{22}$

The remaining fits in Figure 4b-e all include a peak for $\mathrm{C}-\mathrm{O}_{\mathrm{x}}$ and demonstrate the effect of constraining the peak area using different assumptions. By measuring $\mathrm{CO}_{2}$ gas phase spectra, relative sensitivity factors for the $\mathrm{C}: \mathrm{O}$ area ratio were established. (See Supporting Information). Based on the previous discussion of $\mathrm{C} 1 \mathrm{~s}$ binding energies, it is likely that each carbon atom accounted for in the peaks near $287 \mathrm{eV}$ and $288 \mathrm{eV}$ is associated with one or two oxygen atoms. If we add a peak for these $\mathrm{C}-\mathrm{O}_{\mathrm{x}}$ species and assume the maximum of $2 \mathrm{O}$ atoms per $\mathrm{C}$ atom while allowing the peak position to vary in a reasonable range, we obtain the fit shown in Figure $4 \mathrm{~b}$. The 
difference spectrum between the data points and the total fit show clearly that some intensity (shaded) is not accounted for by the $\mathrm{C}-\mathrm{O}_{\mathrm{x}}$ peak in the region between $533-534 \mathrm{eV}$, which is exactly the region where we expect molecular water to appear.

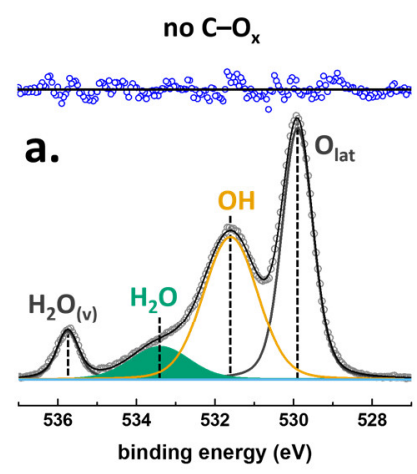

10 per $\mathrm{C}$

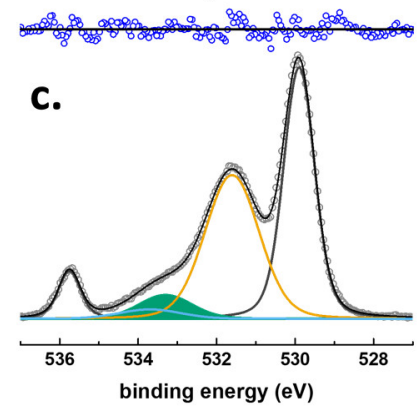

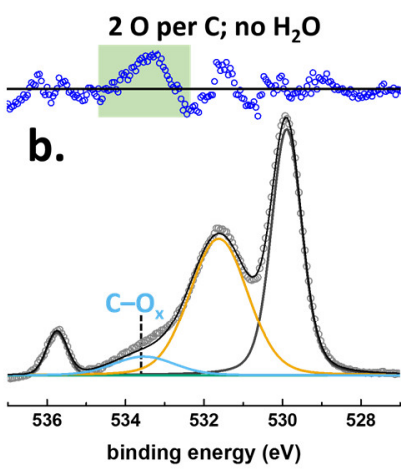

20 per C

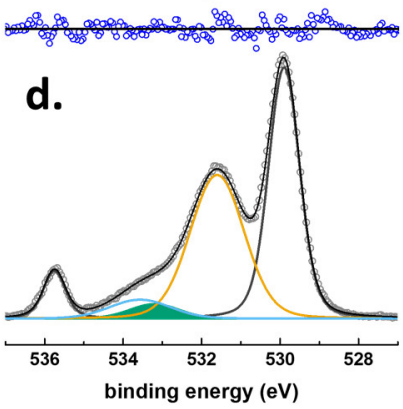

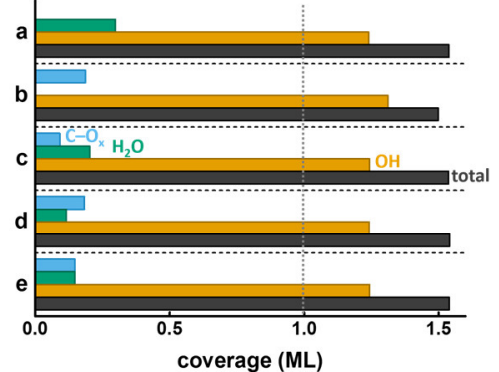

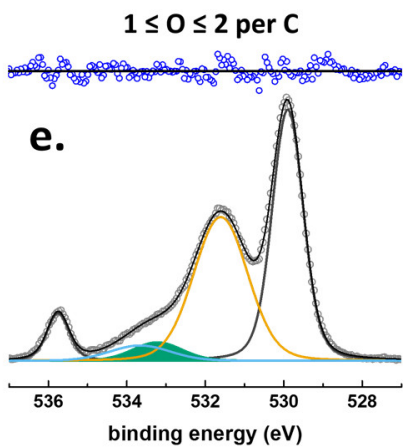

Figure 4. Fits of a single $\mathrm{O} 1 \mathrm{~s}$ spectrum $\left(\mathrm{CuO}, 20{ }^{\circ} \mathrm{C}, 0.1\right.$ Torr water vapor) demonstrating the effect of changing the assumptions made about the quantity of oxidized carbon contaminants. (a) No peak is included for carbon contaminants, leaving only adsorbed molecular water (shaded peak) and gas-phase water $\left(\mathrm{H}_{2} \mathrm{O}_{(\mathrm{v})}\right)$ above $533 \mathrm{eV}$. (b) The area of the $\mathrm{C}-\mathrm{O}_{x}$ peak is fixed, assuming $2 \mathrm{O}$ atoms for each $\mathrm{C}$ atom. The difference spectrum at the top shows that the fit is inadequate, indicating that an additional peak is required for adsorbed molecular water. (c) The area of the $\mathrm{C}-\mathrm{O}_{\mathrm{x}}$ peak is fixed, assuming $1 \mathrm{O}$ atom per $\mathrm{C}$ atom, and a peak for adsorbed molecular water is included. (d) The area of the $\mathrm{C}-\mathrm{O}_{\mathrm{x}}$ peak is fixed, assuming $2 \mathrm{O}$ atoms per $\mathrm{C}$ atom. (e) The area of the $\mathrm{C}-\mathrm{O}_{x}$ peak is allowed to vary during fitting in the range between $1-2 \mathrm{O}$ atoms per $\mathrm{C}$ atom. Note that the difference spectra for (a) and (c-e) are nearly identical. The bar graph in the upper right corner compares calculated coverages in monolayers (ML) of surface species for each fit condition (a-e). Depending on the assumptions made, the coverage of molecular water can vary by more than a factor of 2 .

Figures 4 c-e show fits when an additional peak for water is included and the $\mathrm{C}-\mathrm{O}_{\mathrm{x}}$ peak area is given different constraints. All three of these fits, as well as the fit in Figure 4a, give nearly identical difference spectra. In Figure 4c, only one $\mathrm{O}$ atom per $\mathrm{C}$ atom is assumed, while in Figure 4d, we assumed two $\mathrm{O}$ atoms per $\mathrm{C}$ atom. Figure $4 \mathrm{e}$ shows the best fit where the area of the $\mathrm{C}-\mathrm{O}_{\mathrm{x}}$ 
peak is constrained to be between one and two $\mathrm{O}$ atoms per $\mathrm{C}$ atom. In Figure $4 \mathrm{e}$, the best fit gives an area intermediate of one $\mathrm{O}$ atom per $\mathrm{C}$ atom and two $\mathrm{O}$ atoms per $\mathrm{C}$ atom. This fit is physically and chemically reasonable, as a mixture of carbonaceous species containing one or two oxygen atoms is entirely possible. The bar graph in Figure 4 shows the different surface coverages calculated for $\mathrm{OH}$, water, $\mathrm{C}-\mathrm{O}_{\mathrm{x}}$, and the total surface coverage in monolayers (ML) for each of the fits in Fig. 4a-e. In the following sections, we use the fitting protocol demonstrated in Figure 4e, where the $\mathrm{C}-\mathrm{O}_{\mathrm{x}}$ peak area is constrained to between one and two $\mathrm{O}$ atoms per $\mathrm{C}$ atom, to calculate surface coverages.

The data in Figure 4 demonstrate, however, that an equally good fit can be obtained by assuming no carbon contamination, or the high and low cases of 2:1 and 1:1 O:C atom ratio. When we take into account also the inherent uncertainty in peak fitting and the calculated relative sensitivity factors (on the order of $15-20 \%$ ), the calculated coverages should be taken only as a reasonable approximation based on identification and accounting of the surface carbon contaminants.

\subsection{Effect of the hot ion gauge filament on measurements in the presence of water vapor.}

Pressures in the mTorr range and higher can be measured by absolute capacitance manometers. For pressures up to $1 \times 10^{-4}$ Torr, we require the use of an ion pressure gauge during isotherm measurements. Cracking of gas-phase molecules over the hot metal filament of the gauge should always be considered during experiments, as cracking products may be more reactive than the parent molecule. Here, we demonstrate the difference in surface chemistry as a result of water cracking on the hot pressure-gauge filament.

Figure 5a compares $\mathrm{Cu} 2 \mathrm{p}_{3 / 2}$ spectra collected for $\mathrm{CuO}$ and $\mathrm{Cu}_{2} \mathrm{O}$ using the water leakvalve calibration method described in the experimental section (ion gauge is off during XPS 
measurements). The $\mathrm{Cu} 2 \mathrm{p}_{3 / 2}$ spectra measured at $1 \times 10^{-8}$ Torr and $1 \times 10^{-4}$ Torr are identical. In Figure $5 \mathrm{~b}$, the $\mathrm{Cu} 2 \mathrm{p}_{3 / 2}$ spectrum for $\mathrm{CuO}$ shows distinct changes between these two pressure conditions when the ion gauge is left on. The main peak of the $\mathrm{Cu} 2 \mathrm{p}_{3 / 2}$ region shifts to lower binding energy and narrows, while the satellite features at higher binding energy become smaller. Together, these changes indicate a partial reduction of $\mathrm{Cu}^{2+}$ surface sites to $\mathrm{Cu}^{+}$. In contrast, no changes are observed in the $\mathrm{Cu}_{2} \mathrm{O} \mathrm{Cu} 2 \mathrm{p}_{3 / 2}$ spectrum. The extent of surface hydroxylation also seems to be affected by the ion gauge. In Figure $5 \mathrm{c}$, the hydroxyl coverage at $1 \times 10^{-4}$ Torr is greater when the ion gauge is left on during exposure to water vapor. This trend was consistent across the pressure range measured with the ion gauge on $\left(10^{-8}-10^{-4}\right.$ Torr). For both surfaces, it is clear that the hot filament is reacting with gas phase water and thus subsequently affecting the surface chemistry of the oxides. 

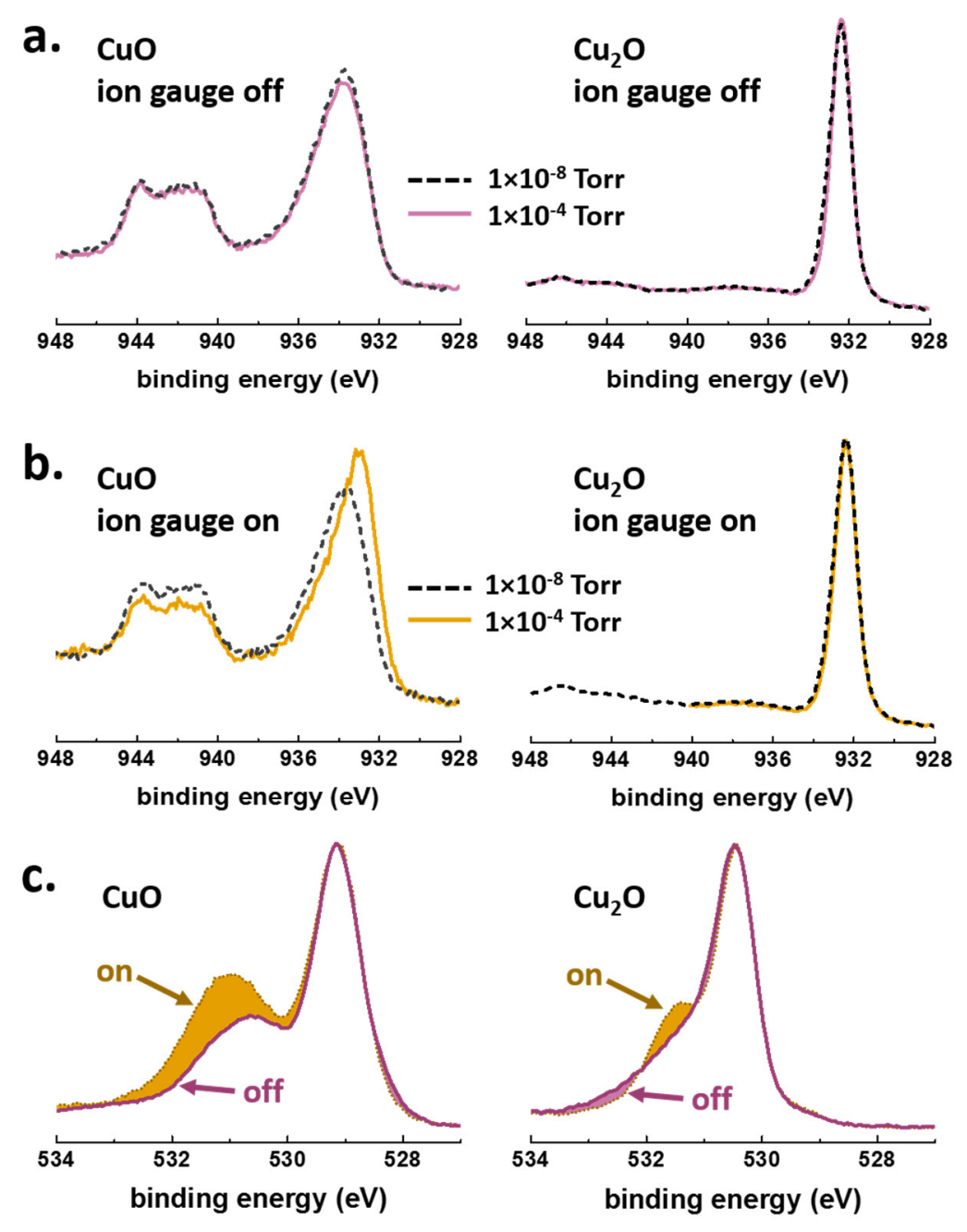

Figure 5. (a-b) $\mathrm{Cu} 2 \mathrm{p}_{3 / 2}$ spectra demonstrating the effect of leaving the ion gauge on during water exposure measurements. In (a), the ion gauge was left off during water exposure, and on both $\mathrm{Cu}_{2} \mathrm{O}$ and $\mathrm{CuO}$, no significant change is observed when the water vapor pressure is increased from $1 \times 10^{-8}$ Torr to $1 \times 10^{-4}$ Torr. In (b), the ion gauge was turned on during water exposure, and while no change is observed for $\mathrm{Cu}_{2} \mathrm{O}$ when the water vapor pressure is increased, the $\mathrm{CuO}$ spectra indicate partial reduction of $\mathrm{Cu}^{2+}$ to $\mathrm{Cu}^{+}$. (c) $\mathrm{O}$ 1s spectra collected at a water vapor pressure of $1 \times 10^{-4}$ Torr showing that the amount of hydroxyl groups on the surface is higher on both $\mathrm{CuO}$ and $\mathrm{Cu}_{2} \mathrm{O}$ when the ion gauge is left on during water exposure. The shaded regions highlight differences between the on and off conditions.

\subsection{Comparison of isobar and isotherm experiments.}

Controlling the relative humidity at the sample surface can be done under constant pressure or constant temperature conditions, and each of these methods has advantages and disadvantages. 
For isotherm measurements, much lower RH conditions can be accessed by beginning at UHV and slowly increasing the water vapor pressure. This approach can be helpful for determining the initial onset of surface hydroxylation, which for many oxide surfaces occurs even under UHV conditions due to reaction with low levels of water vapor in the background. ${ }^{3,8}$ Isotherm measurements also avoid problems associated with chemical changes to the surface due to decomposition of the substrate at elevated temperatures. On the other hand, the adventitious carbon contaminates the surface more quickly in isotherm experiments, particularly at higher water vapor pressures where turbo pumps must be isolated and pumping is reduced (here, $\geq 1 \times 10^{-3}$ Torr). Mild heating of the substrate can help prevent accumulation of contaminants on the surface, which may block or compete with water for adsorption sites, thus making isobar experiments where the sample starts hot and is gradually cooled typically "cleaner" than isotherms collected over the same RH range.

Figure 6 compares one isobar and one isotherm dataset each for $\mathrm{CuO}$ and $\mathrm{Cu}_{2} \mathrm{O}$. During isobar experiments where the sample is heated, the hydroxyl coverage is typically lower than that measured at room temperature under the same $\mathrm{RH}$. This trend holds until higher $\mathrm{RH}(>\sim 0.1 \% \mathrm{RH})$ values are reached.

When isobar and isotherm measurements agree with each other, one can assume equilibrium conditions for water adsorption/desorption. When isobar and isotherm data do not converge, this mismatch could be caused by deviations of the true RH at the sample surface from calculated values in isobar experiments, where the gas phase temperature $T_{\text {gas }}$ is in general lower than that of the surface $\left(T_{\text {surf }}\right.$ ), leading to lower collision frequencies in adsorption. This situation can be taken into account by using an effective water vapor pressure $p^{\prime}=p\left(T_{\text {gas }} / T_{\text {surf }}\right)^{1 / 2}$ for the calculation of the RH, as described in Ref. 12. The RH scale in Figure 6 was calculated without using this correction since the actual gas phase temperature in the very vicinity of the sample is 
not known. The deviation of isobar and isotherm data in Figure 6 is, however, not due to calculation of the $\mathrm{RH}$ for the isobar data, which would give an underestimate of the true $\mathrm{RH}$ and thus produce the opposite effect of that observed in Figure 6. The difference in isobar and isotherm measurements in this case also cannot be due to a difference in total water exposure (in Langmuir). Even though the isotherm experiments have a longer time between each measurement than the isobar experiments, the total water vapor exposure (in Langmuir, L) in isobar experiments is still higher due to the much larger partial pressure of water vapor (e.g. at $\sim 5 \times 10^{-4} \% \mathrm{RH}$ : isotherm, 15 $\min , 9 \times 10^{4} \mathrm{~L}$; isobar, $2 \mathrm{~min}, 1.2 \times 10^{7} \mathrm{~L}$ ). There also appears to be no consistent correlation between the difference in $\mathrm{OH}$ coverage and the amount of carbon contamination. In the case of $\mathrm{CuO}$, there is indeed a higher coverage of $\mathrm{C}-\mathrm{O}_{\mathrm{x}}$ in isotherm experiments where the $\mathrm{OH}$ coverage is higher. On the other hand, the $\mathrm{C}-\mathrm{O}_{\mathrm{x}}$ coverage on $\mathrm{Cu}_{2} \mathrm{O}$ is not always lower for isotherm experiments, and the $\mathrm{OH}$ gap between the isobar and isotherm curves is still prominent.

One possibility is that for the isobar measurements, the lower RH data points are collected when the sample is hot, and some reorientation of the exposed crystal facets at the sample surface could occur upon heating. It has been shown that for many metals and metal oxides, including $\mathrm{Cu}$ and $\mathrm{TiO}_{2}$, changing the exposed crystal facet can have a marked effect on water adsorption and dissociation. ${ }^{8,32,33}$ The effects of crystallographic surface termination is a point which requires further investigation for these and other polycrystalline samples. 

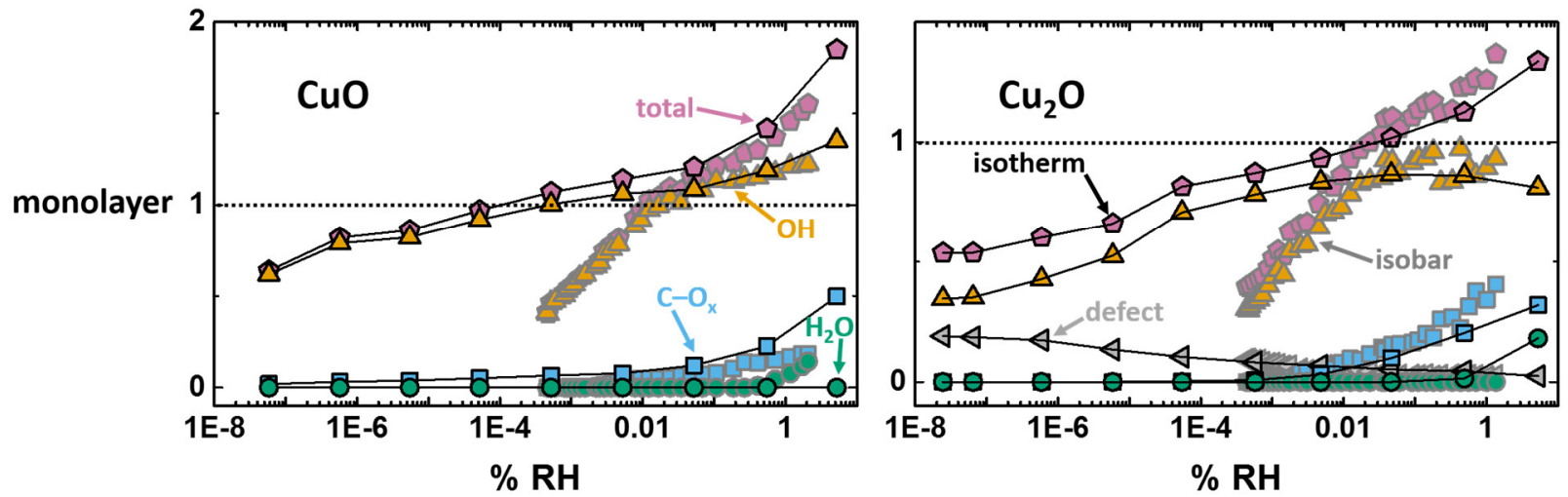

Figure 6. Comparison of isobar $(0.1$ Torr water vapor pressure, grey outlined symbols $)$ and isotherm $\left(20^{\circ} \mathrm{C}\right.$, symbols connected by black lines) water exposure experiments for polycrystalline $\mathrm{CuO}$ and $\mathrm{Cu}_{2} \mathrm{O}$ surfaces. Below $\sim 0.01 \% \mathrm{RH}$, the isotherm measurements show significantly higher $\mathrm{OH}$ coverages on both oxides. This $\mathrm{OH}$ gap between the isobar and isotherm curves may be due to differences in the crystallite orientations when the samples are held at higher temperatures.

In isobar experiments, the sample surfaces are expected to remain cleaner at a given $\mathrm{RH}$ condition, as the adventitious carbon species are less likely to react with the surface before water vapor is introduced. The data in Figure 6 demonstrate that the $\mathrm{CuO}$ samples consistently show lower coverages of $\mathrm{C}-\mathrm{O}_{\mathrm{x}}$ species for isobar compared to isotherm measurements. However, we did not observe a similar trend for the $\mathrm{Cu}_{2} \mathrm{O}$ samples, and sometimes, as shown in Figure 6, the isobar experiments showed slightly higher $\mathrm{C}-\mathrm{O}_{\mathrm{x}}$ coverages. In general, $\mathrm{C}-\mathrm{O}_{\mathrm{x}}$ species were first observed at higher $\mathrm{RH}$ on $\mathrm{Cu}_{2} \mathrm{O}$ than on $\mathrm{CuO}$, and only at $\mathrm{RH}$ values where the partial pressure of water vapor required reduced pumping to the XPS analysis chamber (i.e. above $1 \times 10^{-4}$ Torr). The higher $\mathrm{RH}$ required for onset of $\mathrm{C}-\mathrm{O}_{\mathrm{x}}$ adsorption on $\mathrm{Cu}_{2} \mathrm{O}$, even for similarly low background concentrations of adventitious carbon, suggests that $\mathrm{CuO}$ has a higher affinity for these species than $\mathrm{Cu}_{2} \mathrm{O}$. On both surfaces, there is a correlation between lower $\mathrm{C}-\mathrm{O}_{\mathrm{x}}$ coverage and higher coverages of molecular water. These data therefore suggest that the presence of adventitious carbon contaminants suppresses molecular water adsorption on these surfaces. However, the range of RH values accessible in this study has an upper limit just at the point where molecular water is 
observed. Additional experiments with a wider RH window would help to clarify this point, but would require a method to cool the sample to lower temperatures than were available in our experimental setup. Since the preparation of the $\mathrm{CuO}_{x}$ surfaces requires heating in $\mathrm{O}_{2}$ in the end station, a method for being able to both heat and significantly cool the sample (such that the sample is the coldest point in the chamber) would be required. Qualitatively, both of the copper oxide surfaces appear to have a higher affinity for carbon contaminants than other metal oxides we have studied, and the different $\mathrm{C}$ 1s binding energies measured on the two different oxides suggest that the same background contaminants may undergo different reactions depending on the copper oxidation state. Thus, at least in the case of the copper oxides, the reactivity of the surface with environmental background carbon should be taken into account when considering possible mechanisms of water adsorption and dissociation.

When considering the effects of both adventitious surface carbon and water cracking over the ion-gauge filament, one can build a convincing case for isobar measurements that start with a hot sample as the preferred method for comparing these two oxide surfaces side-by-side. In this way, the presence of surface carbon species, while not completely unavoidable, are at least partially controlled, and we can more comfortably assume that the surface hydroxylation is primarily a result of interactions with molecular water rather than other, more reactive gas-phase species.

Figure 7 shows sets of isobar data for $\mathrm{CuO}$ and $\mathrm{Cu}_{2} \mathrm{O}$. Both surfaces show that in the region between $\sim 0.01-0.1 \% \mathrm{RH}$, the hydroxyl coverage reaches approximately $1 \mathrm{ML}$ and then plateaus. The total coverage continues to increase, primarily due to accumulation of surface carbon species. However, in the case of $\mathrm{CuO}$, molecular water absorption is observed, with onset occurring around $1 \% \mathrm{RH}$. No molecular water is observed on $\mathrm{Cu}_{2} \mathrm{O}$ in our isobar experiments. Based on our isotherm 
experiments and those reported previously by Deng et al., ${ }^{22}$ we do expect molecular water accumulation on $\mathrm{Cu}_{2} \mathrm{O}$ at higher $\mathrm{RH}$ values that are outside the scope of the range attainable in our experimental configuration. However, were the $\mathrm{C}-\mathrm{O}_{\mathrm{x}}$ species not accounted for, one could mistakenly calculate $\sim 0.5 \mathrm{ML}$ of molecular water on $\mathrm{Cu}_{2} \mathrm{O}$ and $\sim 0.4 \mathrm{ML}$ on $\mathrm{CuO}$ at $1 \% \mathrm{RH}$. This situation may be the case in the previous work of Deng et al. with $\mathrm{Cu}_{2} \mathrm{O}$, where the issue of carbon contamination is not discussed.22 Taking caution to account for surface carbon shows that the actual surface coverage of molecular water is significantly lower, even when being conservative in calculating the area of the $\mathrm{C}-\mathrm{O}_{\mathrm{x}}$ peak (see Figure 4 and corresponding discussion).
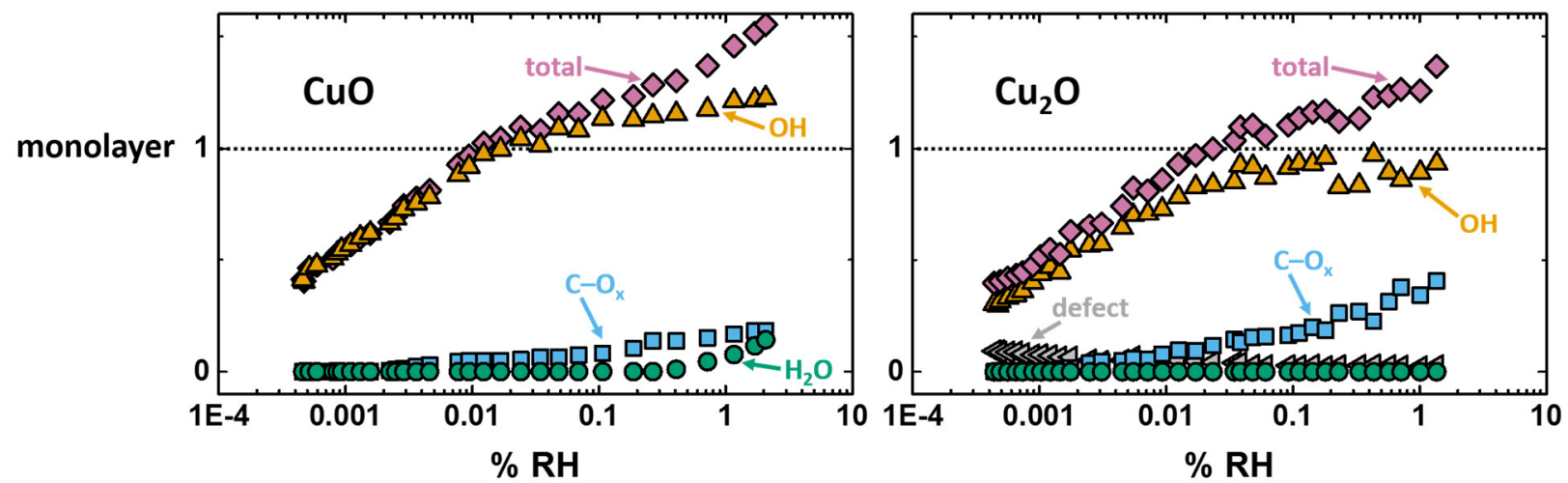

Figure 7. Surface coverage calculations from isobar $\left(p_{\mathrm{H} 2 \mathrm{O}}=0.1\right.$ Torr $)$ measurements on $\mathrm{Cu}_{2} \mathrm{O}$ and $\mathrm{CuO}$. The total coverage of surfaces species was calculated based on attenuation of the $\mathrm{O}_{\text {lat }}$ oxide peak using the procedure outlined in ref. 18 (more detail is available in the Supporting Information). Fractional coverages of other species were calculated by multiplying the total coverage by the fraction of the total peak area corresponding to that species.

\section{Conclusions}

We have studied the initial hydroxylation of copper oxide surfaces in the presence of water vapor. On both $\mathrm{CuO}$ and $\mathrm{Cu}_{2} \mathrm{O}$, the surfaces readily hydroxylated at low relative humidities, with the $\mathrm{OH}$ coverage approaching one monolayer below $0.1 \% \mathrm{RH}$. Molecular water adsorption is only observed once a full monolayer of $\mathrm{OH}$ has formed, which is consistent with general reactivity trends observed on other metal oxide surfaces. Adventitious carbon species also readily adsorb to 
these surfaces, with coverages approaching $0.5 \mathrm{ML}$ on $\mathrm{Cu}_{2} \mathrm{O}$ at $1 \% \mathrm{RH}$, even when precautions are taken to keep the as-prepared surfaces free of carbon. We have demonstrated that quantification of carbonaceous surfaces species is necessary to avoid overestimating the coverage of adsorbed molecular water on these surfaces. We have also demonstrated that cracking of water on the ion gauge filament can affect surface chemistry during APXPS experiments. We found that $\mathrm{CuO}$ surfaces were partially reduced when the ion pressure gauge was turned on during water exposure, and both $\mathrm{CuO}$ and $\mathrm{Cu}_{2} \mathrm{O}$ surfaces hydroxylated more readily. Apart from the technical APXPS considerations detailed here, the initial hydroxylation and hydration of copper oxide surfaces are important chemical processes in numerous technical applications and environmental systems. This work adds to the library of metal oxide surfaces studied by APXPS in order to shed light on inherent reactivity trends of these surfaces under environmentally relevant conditions.

\section{Supporting Information Available}

Supplementary discussions, figures, and tables. This material is available free of charge via the Internet at pubs.acs.org.

\section{Acknowledgments}

This work was supported by the Department of Defense through the Defense Threat Reduction Agency under grant number HDTRA11510005. L.K. acknowledges support from the Danish Research Council for Independent Research and Innovation Fund Denmark (under the National Initiative for Advanced Graphene Coatings and Composites). S.P., H.T., and T.H. acknowledge financial support by Research Foundation - Flanders (FWO) under project number SB-19-151. H.B. acknowledges support by the Director, Office of Science, Office of Basic Energy Sciences, Division of Chemical Sciences, Geosciences, and Biosciences of the US Department of 
Energy under Contract No. DE-AC02-05CH11231. The ALS and the MES beamline 11.0.2 are supported by the Director, Office of Science, Office of Basic Energy Sciences, of the US Department of Energy at the Lawrence Berkeley National Laboratory under Contract No. DEAC02-05CH11231.

References

(1) Head, A. R.; Tang, X.; Hicks, Z.; Wang, L.; Bleuel, H.; Holdren, S.; Trotochaud, L.; Yu, Y.; Kyhl, L.; Karslıoğlu, O. et al. Thermal Desorption of Dimethyl Methylphosphonate from $\mathrm{MoO}_{3}$. Catal. Struct. React. 2017, 3, 112.

(2) Trotochaud, L.; Tsyshevsky, R.; Holdren, S.; Fears, K.; Head, A. R.; Yu, Y.; Karslıoğlu, O.; Pletincx, S.; Eichhorn, B.; Owrutsky, J. et al. Spectroscopic and Computational Investigation of Room-Temperature Decomposition of a Chemical Warfare Agent Simulant on Polycrystalline Cupric Oxide. Chem. Mater. 2017, 29, 7483.

(3) Head, A. R.; Tsyshevsky, R.; Trotochaud, L.; Yu, Y.; Kyhl, L.; Karslıoğlu, O.; Kuklja, M. M.; Bluhm, H. Adsorption of Dimethyl Methylphosphonate on $\mathrm{MoO}_{3}$ : The Role of Oxygen Vacancies. J. Phys. Chem. C 2016, 120, 29077.

(4) Jang, Y. J.; Kim, K.; Tsay, O. G.; Atwood, D. A.; Churchill, D. G. Update 1 Of: Destruction and Detection of Chemical Warfare Agents. Chem. Rev. 2015, 115, PR1.

(5) Yasumasa, T.; Takahiro, N.; Liwei, Y.; Suwilai, C.; Oki, S.; Tomohiro, S.; Tomoya, U.; Mizuki, T.; Yasuhiro, I.; Toshihiko, Y. X-Ray Photoelectron Spectroscopy under Real Ambient Pressure Conditions. Appl. Phys. Express 2017, 10, 076603.

(6) Weatherup, R. S.; Eren, B.; Hao, Y.; Bluhm, H.; Salmeron, M. B. Graphene Membranes for Atmospheric Pressure Photoelectron Spectroscopy. J. Phys. Chem. Lett. 2016, 7, 1622.

(7) Verdaguer, A.; Weis, C.; Oncins, G.; Ketteler, G.; Bluhm, H.; Salmeron, M. Growth and Structure of Water on $\mathrm{SiO}_{2}$ Films on Si Investigated by Kelvin Probe Microscopy and In Situ XRay Spectroscopies. Langmuir 2007, 23, 9699. 
(8) Ketteler, G.; Yamamoto, S.; Bluhm, H.; Andersson, K.; Starr, D. E.; Ogletree, D. F.; Ogasawara, H.; Nilsson, A.; Salmeron, M. The Nature of Water Nucleation Sites on $\mathrm{TiO}_{2}(110)$ Surfaces Revealed by Ambient Pressure X-Ray Photoelectron Spectroscopy. J. Phys. Chem. C 2007, 111,8278 .

(9) Stoerzinger, K. A.; Comes, R.; Spurgeon, S. R.; Thevuthasan, S.; Ihm, K.; Crumlin, E. J.; Chambers, S. A. Influence of $\mathrm{LaFeO}_{3}$ Surface Termination on Water Reactivity. J. Phys. Chem. Lett. 2017, 8, 1038.

(10) Stoerzinger, K. A.; Hong, W. T.; Crumlin, E. J.; Bluhm, H.; Biegalski, M. D.; ShaoHorn, Y. Water Reactivity on the $\mathrm{LaCoO}_{3}$ (001) Surface: An Ambient Pressure X-Ray Photoelectron Spectroscopy Study. J. Phys. Chem. C 2014, 118, 19733.

(11) Deng, X.; Lee, J.; Wang, C.; Matranga, C.; Aksoy, F.; Liu, Z. In Situ Observation of Water Dissociation with Lattice Incorporation at FeO Particle Edges Using Scanning Tunneling Microscopy and X-Ray Photoelectron Spectroscopy. Langmuir 2011, 27, 2146.

(12) Yamamoto, S.; Kendelewicz, T.; Newberg, J. T.; Ketteler, G.; Starr, D. E.; Mysak, E. R.; Andersson, K. J.; Ogasawara, H.; Bluhm, H.; Salmeron, M. et al. Water Adsorption on $\alpha-$ $\mathrm{Fe}_{2} \mathrm{O}_{3}(0001)$ at near Ambient Conditions. J. Phys. Chem. C 2010, 114, 2256.

(13) Deng, X.; Lee, J.; Wang, C.; Matranga, C.; Aksoy, F.; Liu, Z. Reactivity Differences of Nanocrystals and Continuous Films of $\alpha-\mathrm{Fe}_{2} \mathrm{O}_{3}$ on $\mathrm{Au}(111)$ Studied with In Situ X-Ray Photoelectron Spectroscopy. J. Phys. Chem. C 2010, 114, 22619.

(14) Kendelewicz, T.; Kaya, S.; Newberg, J. T.; Bluhm, H.; Mulakaluri, N.; Moritz, W.; Scheffler, M.; Nilsson, A.; Pentcheva, R.; Brown, G. E. X-Ray Photoemission and Density Functional Theory Study of the Interaction of Water Vapor with the $\mathrm{Fe}_{3} \mathrm{O}_{4}(001)$ Surface at NearAmbient Conditions. J. Phys. Chem. C 2013, 117, 2719.

(15) Shavorskiy, A.; Müller, K.; Newberg, J. T.; Starr, D. E.; Bluhm, H. Hydroxylation of Ultrathin $\mathrm{Al}_{2} \mathrm{O}_{3} / \mathrm{NiAl}(110)$ Films at Environmental Humidity. J. Phys. Chem. C 2014, 118, 29340.

(16) Chueh, W. C.; McDaniel, A. H.; Grass, M. E.; Hao, Y.; Jabeen, N.; Liu, Z.; Haile, S. M.; McCarty, K. F.; Bluhm, H.; El Gabaly, F. Highly Enhanced Concentration and Stability of Reactive $\mathrm{Ce}^{3+}$ on Doped $\mathrm{CeO}_{2}$ Surface Revealed In Operando. Chem. Mater. 2012, 24, 1876. (17) Newberg, J. T.; Starr, D. E.; Yamamoto, S.; Kaya, S.; Kendelewicz, T.; Mysak, E. R.; Porsgaard, S.; Salmeron, M. B.; Brown, G. E.; Nilsson, A. et al. Autocatalytic Surface 
Hydroxylation of $\mathrm{MgO}(100)$ Terrace Sites Observed under Ambient Conditions. J. Phys. Chem. C 2011, 115, 12864.

(18) Newberg, J. T.; Starr, D. E.; Yamamoto, S.; Kaya, S.; Kendelewicz, T.; Mysak, E. R.; Porsgaard, S.; Salmeron, M. B.; Brown, G. E.; Nilsson, A. et al. Formation of Hydroxyl and Water Layers on MgO Films Studied with Ambient Pressure Xps. Surf. Sci. 2011, 605, 89. (19) Stoerzinger, K. A.; Hong, W. T.; Azimi, G.; Giordano, L.; Lee, Y.-L.; Crumlin, E. J.; Biegalski, M. D.; Bluhm, H.; Varanasi, K. K.; Shao-Horn, Y. Reactivity of Perovskites with Water: Role of Hydroxylation in Wetting and Implications for Oxygen Electrocatalysis. J. Phys. Chem. C 2015, 119, 18504.

(20) Jribi, R.; Barthel, E.; Bluhm, H.; Grunze, M.; Koelsch, P.; Verreault, D.; Søndergård, E. Ultraviolet Irradiation Suppresses Adhesion on $\mathrm{TiO}_{2}$. J. Phys. Chem. C 2009, 113, 8273.

(21) Mura, A.; Hideshima, I.; Liu, Z.; Hosoi, T.; Watanabe, H.; Arima, K. Water Growth on $\mathrm{GeO}_{2} / \mathrm{Ge}(100)$ Stack and Its Effect on the Electronic Properties of $\mathrm{GeO}_{2}$. J. Phys. Chem. C 2013, $117,165$.

(22) Deng, X.; Herranz, T.; Weis, C.; Bluhm, H.; Salmeron, M. Adsorption of Water on $\mathrm{Cu}_{2} \mathrm{O}$ and $\mathrm{Al}_{2} \mathrm{O}_{3}$ Thin Films. J. Phys. Chem. C 2008, 112, 9668.

(23) Deng, X.; Verdaguer, A.; Herranz, T.; Weis, C.; Bluhm, H.; Salmeron, M. Surface Chemistry of $\mathrm{Cu}$ in the Presence of $\mathrm{CO}_{2}$ and $\mathrm{H}_{2} \mathrm{O}$. Langmuir 2008, 24, 9474.

(24) Bluhm, H.; Hävecker, M.; Knop-Gericke, A.; Kleimenov, E.; Schlögl, R.; Teschner, D.; Bukhtiyarov, V. I.; Ogletree, D. F.; Salmeron, M. Methanol Oxidation on a Copper Catalyst Investigated Using In Situ X-Ray Photoelectron Spectroscopy. J. Phys. Chem. B 2004, 108, 14340.

(25) Jug, K.; Heidberg, B.; Bredow, T. Cyclic Cluster Study of Water Adsorption Structures on the $\mathrm{MgO}(100)$ Surface. Surf. Sci. 2007, 601, 1529.

(26) Jug, K.; Heidberg, B.; Bredow, T. Molecular Dynamics Study of Water Adsorption Structures on the MgO(100) Surface. J. Phys. Chem. C 2007, 111, 6846.

(27) Ogletree, D. F.; Bluhm, H.; Hebenstreit, E. D.; Salmeron, M. Photoelectron Spectroscopy under Ambient Pressure and Temperature Conditions. Nucl. Instrum. Methods Phys. Res., Sect. A 2009, 601, 151.

(28) Bluhm, H.; Andersson, K.; Araki, T.; Benzerara, K.; Brown, G. E.; Dynes, J. J.; Ghosal, S.; Gilles, M. K.; Hansen, H. C.; Hemminger, J. C. et al. Soft X-Ray Microscopy and 
Spectroscopy at the Molecular Environmental Science Beamline at the Advanced Light Source. J. Electron. Spectrosc. Relat. Phenom. 2006, 150, 86.

(29) Jiang, P.; Prendergast, D.; Borondics, F.; Porsgaard, S.; Giovanetti, L.; Pach, E.; Newberg, J.; Bluhm, H.; Besenbacher, F.; Salmeron, M. Experimental and Theoretical Investigation of the Electronic Structure of $\mathrm{Cu}_{2} \mathrm{O}$ and $\mathrm{CuO}$ Thin Films on $\mathrm{Cu}(110)$ Using X-Ray Photoelectron and Absorption Spectroscopy. J. Chem. Phys. 2013, 138, 024704.

(30) Ghijsen, J.; Tjeng, L. H.; van Elp, J.; Eskes, H.; Westerink, J.; Sawatzky, G. A.; Czyzyk, M. T. Electronic Structure of $\mathrm{Cu}_{2} \mathrm{O}$ and CuO. Phys. Rev. B 1988, 38, 11322.

(31) Beamson, G.; Briggs, D. High Resolution XPS of Organic Polymers: The Scienta ESCA300 Database; Wiley, 1992.

(32) Yamamoto, S.; Andersson, K.; Bluhm, H.; Ketteler, G.; Starr, D. E.; Schiros, T.; Ogasawara, H.; Pettersson, L. G. M.; Salmeron, M.; Nilsson, A. Hydroxyl-Induced Wetting of Metals by Water at Near-Ambient Conditions. J. Phys. Chem. C 2007, 111, 7848.

(33) Yamamoto, S.; Bluhm, H.; Andersson, K.; Ketteler, G.; Ogasawara, H.; Salmeron, M.; Nilsson, A. In Situ X-Ray Photoelectron Spectroscopy Studies of Water on Metals and Oxides at Ambient Conditions. J. Phys.: Condens. Matter 2008, 20, 184025. 


\section{O 1s XPS spectra at $\sim 0.001 \% \mathrm{RH}$}
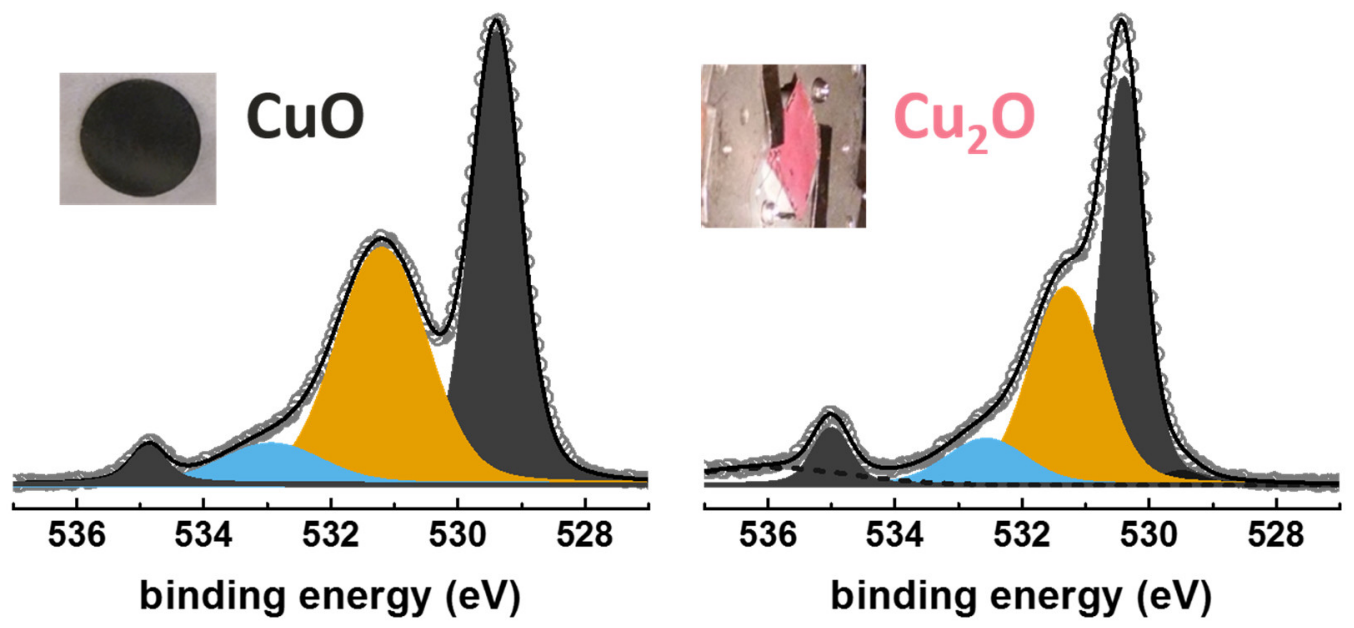